\title{
A Clique-Based Online Algorithm for Constructing Optical Orthogonal Codes
}

\author{
Yuan Zhang ${ }^{\mathrm{a}}$, Mao Peng ${ }^{\mathrm{a}}$, Shengxiang Yang ${ }^{\mathrm{a}, \mathrm{b}, *}$ \\ ${ }^{a}$ College of Mathematics and Statistics, Nanjing University of Information Science and Technology, Nanjing 210044, China \\ ${ }^{b}$ Centre for Computational Intelligence (CCI), School of Computer Science and Informatics, De Montfort University, Leicester LE1 9BH, United Kingdom
}

\begin{abstract}
An optical orthogonal code (OOC) is a family of binary sequences with good auto- and cross-correlation properties. In the literature, various mathematical tools have been used to construct OOCs with specific parameters. But, to find a complete solution for constructing OOCs with an arbitrary setting of parameters is still difficult at the moment. In this paper, a clique-based online algorithm is proposed to construct OOCs of relatively large sizes. In the proposed algorithm, the construction of OOCs is reduced to the maximum clique problem based on specially generated graphs, where vertices represent the codewords of an OOC and edges represent the cross-correlation relationships between codeword pairs. In order to overcome the limitation of computer memory for storing large graphs, part of the graph vertices are supposed to arrive sequentially to be fed into the proposed algorithm, and a specially designed evolutionary algorithm is used to find the maximum clique of the current graph when new vertices arrive. The proposed algorithm does not use parameter-specific techniques and hence can be used for different code weight and correlation constraints. Experiments show that the proposed algorithm outperforms an offline evolutionary algorithm with guided mutation on constructing OOCs.
\end{abstract}

Keywords: Optical orthogonal codes, evolutionary algorithm, maximum clique problem, online algorithm

\section{Introduction}

Code division multiple access (CDMA) is one of the most attractive multiple-access schemes for optical communication networks because it allows simultaneous users to access the same optical channel asynchronously with no delay or scheduling. In optical CDMA systems, each user is assigned a unique binary signature sequence (or codeword) as its own address. A user's receiver must be able to extract its signature sequence in the presence of other users' signature sequences. Therefore, a set of signature sequences that are distinguishable from time-shifted versions of themselves and for which any two such signature sequences are easily distinguishable from (a possible time-shifted version of) each other are needed. Researchers in the CDMA domain have proposed several coding systems, such as the prime code [28] and optical orthogonal code (OOC) [31].

The mathematical model fo the OOC can be briefly described as follows. Let $v, k, \lambda_{a}$ and $\lambda_{c}$ be positive integers. A $\left(v, k, \lambda_{a}, \lambda_{c}\right)$-OOC $C$ is a family of $(0,1)$ sequences with length (or order) $v$ and weight $k$ (i.e., the given number of one bits in the sequences) which satisfies the following two properties:

1. The auto-correlation property:

$$
\sum_{t=0}^{v-1} x_{t} x_{t \oplus \tau} \leq \lambda_{a}
$$

\footnotetext{
${ }^{*}$ Corresponding author.

Email addresses: zhangyuanppx@163.com (Yuan Zhang), mpengsjtu@gmail.com (Mao Peng), syang@dmu.ac.uk (Shengxiang Yang)
}

for any $\mathbf{x} \in C$ and any integer $\tau(0<\tau<v)$, where " $\oplus$ " represents the modulo $v$ addition.

2. The cross-correlation property:

$$
\sum_{t=0}^{v-1} x_{t} y_{t \oplus \tau} \leq \lambda_{c}
$$

for any $\mathbf{x} \neq \mathbf{y}(\mathbf{x}, \mathbf{y} \in \mathcal{C})$ and any integer $\tau(0<\tau<v)$.

The numbers $\lambda_{a}$ and $\lambda_{c}$ are called the auto- and crosscorrelation constraints, respectively. $\mathrm{A}\left(v, k, \lambda_{a}, \lambda_{c}\right)$-OOC with $\lambda_{a}=\lambda_{c}=\lambda$ is called "symmetric" and can be briefly denoted by $(v, k, \lambda)$-OOC. The high weight of codewords facilitates the detection of desired signals, and the low auto- and cross-correlations reduce the interference from unwanted signals of synchronization in a network. An example of an (11, 4, 2)-OOC is given later in Section 2.1 .

Experiments have shown that the setting of OOC's autocorrelation and cross-correlation constraints guarantees a large number of asynchronous users to transmit information efficiently and reliably in a communication network. In short, the auto-correlation of each sequence in the OOC exhibits the thumbtack shape, and the cross-correlation between any two sequences remains low throughout. The lack of a network synchronization requirement enhances the flexibility of OOCbased systems [31,23]. In addition to the applications in optical multiple-access channels, OOCs also find applications in areas such as mobile radio and radar and sonar signal design. For more details, readers are referred to the survey paper by Colbourn et al. [16].

One of the central problems in the OOC research is to determine an OOC's maximum code size, which is the number of 
codewords in the OOC. The code size of an OOC implies the possible number of users in a communication network. In the literature, various mathematical tools have been used to construct OOCs with specific parameters (usually of small sizes) $[1,2,9,11]$. But, it is still a long way to go to find a complete solution for constructing OOCs with an arbitrary setting of parameters (i.e., $k, \lambda_{a}$, and $\lambda_{c}$ ).

In this paper, a clique-based online algorithm is proposed to construct OOCs with relatively large sizes. The proposed algorithm is based on specially generated graphs, where vertices represent the codewords of an OOC that satisfy the autocorrelation constraint and edges represent the cross-correlation relationships between codeword pairs. Based on such specially generated graphs, the construction of OOCs is reduced to the maximum clique problem (MCP). In order to overcome the limitation of computer memory for storing large graphs, part of the graph vertices are supposed to arrive sequentially to be fed into the proposed algorithm, and a specially designed evolutionary algorithm (EA) is used to find the maximum clique of the current graph when new vertices arrive. The proposed algorithm is tested to design $(v, 4,2)$-OOCs. Experimental results show that the cardinalities of constructed OOCs are close to the Johnson bound (to be described in Section 2.2) when $v$ is small. We also use the proposed algorithm to construct $(v, 4,2,1)$-OOCs and $(v, 5,2)$-OOCs, indicating that the proposed algorithm can be used under different settings of parameters.

The rest of this paper is organized as follows. Section 2 describes the background and related work on OOCs, including an example of an OOC, the fundamentals of constructing OOCs and a brief review of search algorithms for constructing OOCs. Our proposed clique-based online algorithm for constructing OOCs is presented in Section 3. Experimental results are reported in Section 4. Finally, Section 5 concludes this paper with some discussions on relevant future work.

\section{Background and Related Work}

\subsection{Example of an $\mathrm{OOC}$}

In order to better understand the concept of OOCs, we give an example here. Let $C$ be a code of length $v=11$ and weight $k=4$ with three codewords $C_{1}, C_{2}, C_{3}$ as follows:

$$
C=\left\{C_{1}, C_{2}, C_{3}\right\}=\{11000010010,11000101000,11101000000\} .
$$

In order to calculate the auto-correlation constraint of $C$, we take $C_{1}=11000010010$ for instance. Its 11 cyclic shifts $C_{1 i}$ $(i=0,1, \cdots, 10)$ are given as follows:

$$
\begin{aligned}
& C_{1,0}=11000010010, C_{1,1}=01100001001, \\
& C_{1,2}=10110000100, C_{1,3}=01011000010, \\
& C_{1,4}=00101100001, C_{1,5}=10010110000, \\
& C_{1,6}=01001011000, C_{1,7}=00100101100, \\
& C_{1,8}=00010010110, C_{1,9}=00001001011, \\
& C_{1,10}=10000100101 .
\end{aligned}
$$

The inner product of any pair from these shifts is no more than 2 , which indicates the auto-correlation constraint of $C_{1}$ is 2 .
Similarly, we can check that the auto-correlation constraint of $C_{2}$ and $C_{3}$ is also 2. Hence, $C$ has an auto-correlation constraint of 2, i.e., $\lambda_{a}=2$.

In order to work out the cross-correlation constraint of $C$, we first obtain all the cyclic shifts of all codewords in $C$, i.e., $C_{i l}$ ( $i \in\{1,2,3\}$ and $l \in\{0,1, \cdots, 10\})$, as shown in Eqs. (4), (5) and (6), respectively.

$$
\begin{aligned}
& C_{2,0}=11000101000, C_{2,1}=01100010100, \\
& C_{2,2}=00110001010, C_{2,3}=00011000101, \\
& C_{2,4}=10001100010, C_{2,5}=01000110001, \\
& C_{2,6}=10100011000, C_{2,7}=01010001100, \\
& C_{2,8}=00101000110, C_{2,9}=00010100011, \\
& C_{2,10}=10001010001 . \\
& C_{3,0}=11101000000, C_{3,1}=01110100000, \\
& C_{3,2}=00111010000, C_{3,3}=00011101000, \\
& C_{3,4}=00001110100, C_{3,5}=00000111010, \\
& C_{3,6}=00000011101, C_{3,7}=10000001110, \\
& C_{3,8}=01000000111, C_{3,9}=10100000011, \\
& C_{3,10}=11010000001 .
\end{aligned}
$$

Then, we calculate the inner product of any pair of shifts from different $C_{i}$ s, i.e., any pair $\left(C_{i l}, C_{j m}\right)(i, j \in\{1,2,3\}$ and $i \neq j$, and $l, m \in\{0,1, \cdots, 10\})$. It is not hard to check that the maximal inner product is 2 . Hence, the cross-correlation constraint of $C$ is 2, i.e., $\lambda_{c}=2$.

In summary, $C$ is a $(11,4,2)$-OOC with 3 codewords.

\subsection{Constructing OOCs: Fundamentals}

As aforementioned, one of the central problems in the OOC research is to determine the code size of an OOC, i.e., the number of codewords in an OOC. An OOC is called maximal if there is no other OOC of a larger size with the same set of parameters. This maximal code size is denoted by $\Phi\left(v, k, \lambda_{a}, \lambda_{c}\right)$, which can be abbreviated by $\Phi(v, k, \lambda)$ when the OOC is symmetric. It is well-known that the size of a $(v, k, \lambda)$-OOC cannot exceed the so-called Johnson bound [15], which is given as follows:

$$
J(v, k, \lambda)=\left\lfloor\frac{1}{k}\left\lfloor\frac{v-1}{k-1} \cdots\left\lfloor\frac{v-\lambda}{k-\lambda}\right\rfloor \cdots\right\rfloor\right\rfloor .
$$

where $\lfloor X\rfloor$ is the floor function, which returns the maximum integer that is less than or equal to $X$.

Usually, a $\left(v, k, \lambda_{a}, \lambda_{c}\right)$-OOC is said to be optimal when its size reaches an upper bound deducible from very general considerations which are valid for all possible pairs $(v, k)$ (with $\lambda_{a}$ and $\lambda_{c}$ fixed). Thus, an optimal OOC is maximal, but the reverse is not true generally.

In the past years, different researchers have devoted effort to the search for maximal or optimal OOCs, and many good results have been obtained by an extensive use of various mathematical tools, such as the design theory [9], difference family [1], relative difference family [11], and projective geometry [2], etc. For example, the existence problem for an optimal $(v, k, 1)$ OOC has been completely solved for $k=3$ [9, 15]. But, it is still difficult to find a complete solution for larger values of $k$, $\lambda_{a}$, and $\lambda_{c}$ for the moment. For example, only some partial answers have been achieved for the cases of $k=4$ or $5, \lambda_{a} \leq 2$ 
and $\lambda_{c} \leq 2[2,3,12,13,14,18]$. Further details can be found in the references and therein.

Since it is still a long way to find a complete solution for constructing OOCs with an arbitrary setting of parameters $k, \lambda_{a}$ and $\lambda_{c}$, in this paper we investigate the construction of OOCs by using search algorithms. Note that we are not aiming to find maximal OOCs, but to present a general scheme to find OOCs with relatively large sizes.

\subsection{Search Algorithms for Constructing OOCs}

The algorithm proposed in this paper is based on graph cliques: all the codewords that satisfy the auto-correlation constraint are viewed as vertices of a graph, and edges are added to the codeword pairs if the cross-correlation constraints are satisfied. For a simple undirected graph $G=(V, E)$ with the vertex set $V$ and edge set $E$, a subset $S$ of $V$ is a clique of $G$ if every two distinct vertices of $S$ are joined by an edge. Naturally, the maximal OOC construction problem can be transformed to the MCP.

The MCP is one of the well-known problems in graph theory, and is related to many real-world problems, such as in social networks, computer vision, computational biochemistry and bio-informatics. In past decades, different exact algorithms have been proposed to achieve maximum cliques, such as branch-and-bound [26] and branch-and-cut [30]. On the other hand, it has been proved that there is no polynomial-time algorithm for approximating the maximal clique within a factor of $n^{1-\varepsilon}$ for any positive $\varepsilon$ unless $P=N P$ [21], where $n$ is the number of vertices in the graph. As a result, it seems to be impossible to have an exact polynomial-time algorithm for solving the MCP. Hence, there has been a lot of researches on using heuristic algorithms for solving the MCP, including local search [8, 22], greedy algorithms [20, 27], constraint programming [29], simulated annealing [19], and EAs [7, 10, 33]. For more details, readers are referred to a recent survey by $\mathrm{Wu}$ and Hao [32].

Although there are many good algorithms for solving the MCP, clique-based algorithms have not been frequently used to design OOCs. To the best of our knowledge, it seems that the only existing clique-based algorithm is the one proposed by Chu and Colbourn [14]. In [14], a clique-based algorithm was presented to construct $(v, 4,2)$-OOCs when $v$ is small, and the clique solvers were a reactive local search algorithm from Battiti and Protasi [8] and an exact branch-and-bound algorithm developed by Niskanen and Ostergard [26]. Although the OOCs obtained in [14] are optimal, some parameter-specific techniques were used to accelerate graph generation. As a result, the method can not be generalized to construct OOCs with an arbitrary setting of parameters. More importantly, with the growth of the OOC order, the number of codewords satisfying the auto-correlation condition would become huge.

For example, we have used a heuristic algorithm to test the lower bounds of the graph cardinality for $(v, 4,2)-,(v, 5,2)-$ and $(v, 6,2)$-OOCs (the details will be given in Section 4.2 later). The results show that the cardinality for $(300,4,2)-O O C$ is already over one million. In this case, even storing the graph structure in the computer memory is difficult. This may be the reason why authors in [14] chose to construct optimal $(v, 4,2)$ OOCs for $v$ less than 45 .

Apart from clique-based algorithms, researchers have also used backtracking to search difference sets which would lead to OOCs. Recently, Baicheva and Topalova [4, 5] developed a parallel backtrack search algorithm to find $(v, 4,2,1)$ - and $(v, 5,2,1)$-OOCs when $v$ is small. Such a parallel algorithm was run over IBM Blue Gene supercomputers, and returned optimal OOCs. However, it is not hard to derive from their papers that the reported backtracking is equivalent to clique search. When $v$ is large, the corresponding graph structure still can not be stored in supercomputers. Moreover, the backtracking uses parameter-specific techniques to speedup vertex selection. So, it can not be generalized to construct OOCs with $\lambda_{c} \neq 1$.

In this paper, we propose a clique-based online algorithm to construct OOCs with relatively large sizes. In our setting, the vertices are supposed to arrive sequentially, instead of being available all at once. This way, we can build a pool to store part of the vertices, which is to overcome the limitation of computer memories. A prespecified substitution technique is used to update candidate solutions. Note that the vertices of OOC graphs are generated without using any parameter-specific techniques, which means our algorithm can be used for an arbitrary setting of code weight and correlation constraints.

\section{Proposed Clique-Based Online Algorithm}

In this section, we present the clique-based online algorithm to find large $\left(v, k, \lambda_{a}, \lambda_{c}\right)$-OOCs for general setting of $k, \lambda_{a}$ and $\lambda_{c}$. Since the construction of maximal OOCs can be reduced to the MCP, we first need a method to generate the needed graph. Then, we will run an online algorithm to find cliques, where the so-called Repair and Evolution operations will be used frequently. After the target clique is obtained, a required OOC will follow accordingly.

\subsection{Representation of OOC Codewords}

As mentioned before, a $\left(v, k, \lambda_{a}, \lambda_{c}\right)$-OOC is a family of binary strings with length $v$ and weight $k$. For practical reasons, $v$ is often much bigger than $k$, and this would bring many zeros in each of the codeword string. In this case, a set-theoretic way can be considered to represent codewords.

$\mathrm{A}\left(v, k, \lambda_{a}, \lambda_{c}\right)$-OOC $C$ can be considered as a family of $k$-sets of integers modulo $v$ in which each $k$-set corresponds to a codeword and the integers within each $k$-set specify the non-zero bits. The correlation properties in this set-theoretic framework are shown as follows:

1. The auto-correlation property:

$$
\left|C_{i} \cap\left(C_{i} \oplus \tau\right)\right| \leq \lambda_{a}
$$

for any $C_{i} \in C$ and any integer $\tau(0<\tau<v)$, where " $\oplus$ " represents the modulo $v$ addition.

2. The cross-correlation property:

$$
\left|C_{i} \cap\left(C_{j} \oplus \tau\right)\right| \leq \lambda_{c}
$$

for any $C_{i}, C_{j} \in C(i \neq j)$ and any integer $\tau(0<\tau<v)$. 
Table 1: Equivalent codewords of codeword $\{0,1,6,9\}$

\begin{tabular}{c|cccc}
\hline Codeword & $\{0,1,6,9\}$ & $\{1,2,7,10\}$ & $\{2,3,8,0\}$ & $\{3,4,9,1\}$ \\
\hline Sorted & $\{0,1,6,9\}$ & $\{1,2,7,10\}$ & $\{0,2,3,8\}$ & $\{1,3,4,9\}$ \\
Key & 196 & 1660 & 283 & 1747 \\
\hline Codeword & $\{2,4,5,10\}$ & $\{0,3,5,6\}$ & $\{6,7,1,4\}$ & $\{7,8,2,5\}$ \\
\hline Sorted & $\{4,5,10,2\}$ & $\{5,6,0,3\}$ & $\{1,4,6,7\}$ & $\{2,5,7,8\}$ \\
Key & 3211 & 424 & 1888 & 3352 \\
\hline Codeword & $\{8,9,3,6\}$ & $\{9,10,4,7\}$ & $\{10,0,5,8\}$ & \\
\hline Sorted & $\{3,6,8,9\}$ & $\{4,7,9,10\}$ & $\{0,5,8,10\}$ & \\
Key & 4816 & 6280 & 703 & \\
\hline \multicolumn{5}{|c}{}
\end{tabular}

Throughout this paper, we will use the set vector form $\left(c_{1}, c_{2}, \cdots, c_{k}\right)$ to denote a codeword. Let us use the $(11,4,2)$ OOC in Eq. (3) as an example. In the set notation, it can be represented as follows:

$$
C=\{\{0,1,6,9\},\{0,1,5,7\},\{0,1,2,4\}\} .
$$

For the sake of convenience, a codeword can also be represented by a new variable Key as follows:

$$
K e y=\sum_{i=1}^{k} c_{i} v^{k-i}
$$

where $c_{1}<c_{2}<\cdots<c_{k}$. This way, we can easily get a new codeword out of a block vector $C \in C$ with a shift $C+t$, and we call $C$ and its shift $C+t$ equivalent. Note that equivalent codewords can not be included in one OOC, in order to distinguish inequivalent codewords. We define the characteristic of a codeword $C$ by the minimum Key of the codewords equivalent to $C$.

Taking the codeword $\{0,1,6,9\}$ mentioned above for instance, its equivalent codewords are shown in Table 11 Obviously, the Key value 196 of codeword $\{0,1,6,9\}$ is the smallest among the equivalent codewords. So, we can say the characteristic of codeword $\{0,5,8,10\}$ is 196 since it is equivalent to codeword $\{0,1,6,9\}$.

\subsection{Generation of OOC Graphs}

A graph is an ordered pair $G=(V, E)$ comprising a set $V$ of vertices together with a set $E$ of edges. For the case of an OOC, all the codewords meeting the auto-correlation condition make the vertex set, and edges are added to the codeword pairs if the cross-correlation constraints are satisfied.

In order to accelerate the search of OOC vertices, we can use the following isomorphism technique. If we have got one OOC $C$, then we can get some isomorphism OOCs by mapping $C$ to $t \cdot C$, where $v$ and $t$ are relative prime numbers, and $t \cdot C=\{t C(\bmod v) \mid C \in C\}$. We call $C$ and $t \cdot C$ multiplier equivalents. For example, let $C$ be the $(11,4,2)-O O C$ $\{\{0,1,6,9\},\{0,1,5,7\},\{0,1,2,4\}\}$, after mapping $C$ to $2 \cdot C$, we can obtain a new $(11,4,2)$-OOC $\{\{0,1,2,7\},\{0,2,3,10\}$, $\{0,2,4,8\}\}$. Since the technique is not parameter-specific, it can be applied for any setting of parameters.

In our algorithm, this multiplier trick is used to get codewords that satisfy the auto-correlation constraint efficiently. If

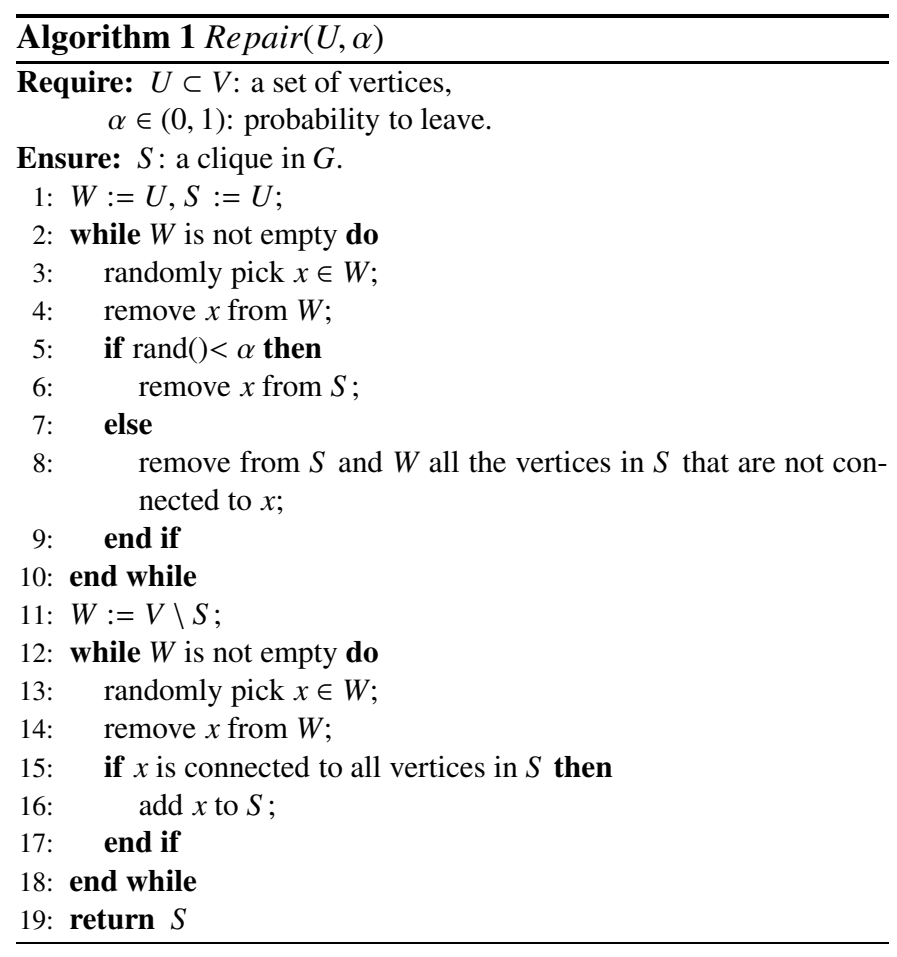

we get a $\left(v, k, \lambda_{a},-\right)$ codeword $C$, we can generate a family of $\left(v, k, \lambda_{a},-\right)$ codewords as follows:

$$
\{t \cdot C \mid \operatorname{gcd}(t, v)=1\}
$$

where $\operatorname{gcd}(t, v)$ returns the greatest common divisor of $t$ and $v$. Note that there might be some equivalent codewords generated. Although there might be some equivalent codewords generated, we can easily distinguish them from the representatives.

\subsection{Clique Repair Operation}

Throughout the running of our online algorithm, we will repeatedly update the current graph and current cliques, and we also need to derive a clique from any subset of vertices. Here, Marchiori's repair technique [24, 25] is used. The main idea is that we first cut some vertices from the graph to get a small clique, and then expand it in a greedy manner until a maximal clique is achieved.

The pseudo-code of the repair operation is shown in Algorithm 1, where $\operatorname{rand}()$ in line 5 returns a uniform random number in the range $[0,1]$. The probability parameter $\alpha \in(0,1)$ used in the first phase must be small; otherwise, the clique returned would be very small.

\subsection{Evolution Operation for the Population of Cliques}

Our proposed algorithm is a clique-based EA. As usual, the EA maintains a population of candidate cliques (solutions). The current candidate population pop $(t)$ is supposed to have $N$ binary strings $X^{i}=\left(x_{1}^{i}, \cdots, x_{n}^{i}\right), i=1,2, \cdots, N$, where $n$ is the number of graph vertices and each string represents a clique. Thus, $p o p(t)$ is an $N \times n$ matrix with elements in $\{0,1\}$. Inspired by the estimation of distribution algorithm (EDA), the 


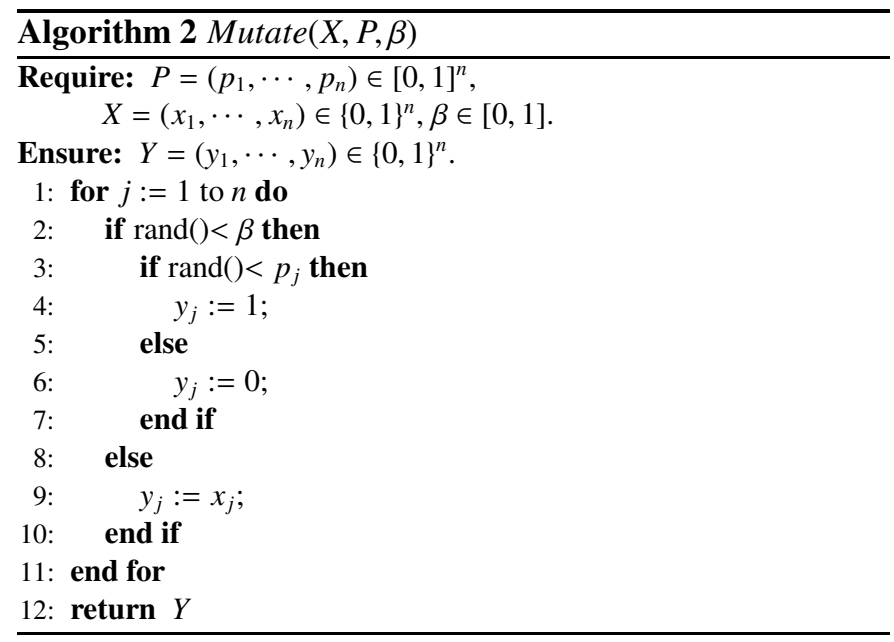

EA maintains a probability vector $P=\left(p_{1}, \cdots, p_{n}\right)$, which is initialized as follows:

$$
p_{j}=\frac{1}{N} \sum_{i=1}^{N} x_{j}^{i} .
$$

At each step, we calculate the fitnes 1 of each binary string in the population, sort the binary strings in the descending order of their fitness, and pick up the fittest $M$ individuals, denoted as $X^{1}, X^{2}, \cdots, X^{M}$. Then, the probability vector $P$ is updated in the same way as in the population-based incremental learning (PBIL) algorithm [6] as follows:

$$
P=(1-\lambda) P+\frac{\lambda}{M} \sum_{i=1}^{M} X^{i}
$$

Then, we apply the guided mutation operator, as shown in Algorithm 2, with the updated probability vector $P$ to string $X^{1}$ for $N-M$ times, repair the resultant strings and use them to replace strings $X^{M+1}, X^{M+2}, \cdots, X^{N}$. The strings $X^{2}, \cdots, X^{M}$ do not generate offspring, but are only used to update the probability vector $P$.

The so-called guided mutation operator is a technique used to combine the global statistical information and local information to overcome the shortcoming of genetic algorithms and EDAs. For the MCP, the guided mutation operator uses the probability vector $P=\left(p_{1}, p_{2}, \cdots, p_{n}\right)$ to mutate an individual $X \in\{0,1\}^{n}$. In detail, for each bit of a binary string that represents an individual, a coin is flipped with a head probability $\beta$. If the head turns up, the specific bit is set to 1 with the probability $p_{i}$; otherwise, it is set to 0. According to the experiments in [33], such a guided mutation operator leads to a better performance than a number of peer algorithms compared in [33].

For the probability vector $P$, we denote the number of positions satisfying $p_{j}>0.0001$ as $t_{1}$, and the number of positions satisfying $p_{j}>0.8$ as $t_{2}$. If $t_{2} / t_{1} \geq 0.8$, then all the strings

\footnotetext{
${ }^{1}$ The fitness of a solution is defined as the cardinality of the clique represented by the solution.
}

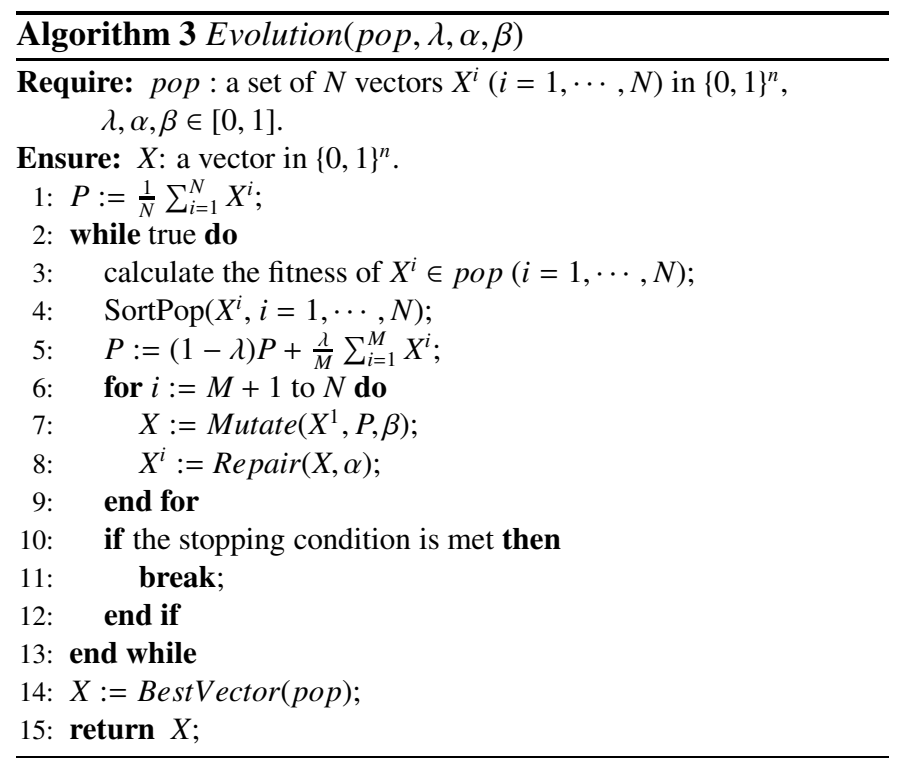

in $p o p(t)$ are thought to be identical, and we return the current best candidate and terminate the evolution operation. The pseudo-code of the whole evolution operation is as shown in Algorithm 3

\subsection{Online Algorithms for the MCP}

In this subsection, we present algorithms for the MCP, which are motivated by the EA with guided mutation $(\mathrm{EA} / \mathrm{G})$ for the MCP [33]. The experimental results reported in [33] show that EA/G performs better than two other EAs, i.e., HGA and MIMIC, which were introduced in [17] and [25] respectively, for solving the MCP.

Since the OOC graphs are often too big, our clique-based algorithms are given under an online setting: the vertices of the graph are supposed to be added one by one sequentially to be fed into the algorithm, instead of having all the vertices available from the start of running the algorithm. Hence, the algorithm is called an online algorithm. Since the whole input is unknown in advance, the online algorithm needs to make decisions that may later turn out not to be optimal. However, it does show efficient performance in real world.

A natural thinking is to re-calculate the clique every time a new vertex arrives, which would take a lot of time. Here, we use a different strategy which stores a pool of candidates that are potential maximum cliques, and then uses the coming vertex to update them. In the following, we develop two algorithms based on such a strategy: one is called EA with Substitution (EAS) for large graphs and another is called EA with Addition (EAA) for medium-sized graphs. For both algorithms, the number of maximal allowed candidates in the pool is set to MaxCache. We use a matrix to store such a pool, each row of which is denoted by $c l q^{i}(i=1, \cdots$, MaxCache $)$.

For very large graphs, it is difficult to store the whole graph in the memory at a time. Let MaxPoint be the maximum cardinality of a graph we can deal with, and denote these MaxPoint points as the static part $S$ of the graph, and the rest as the online part $T$ of the graph. In EAS, we first find a clique of $S$ with a 


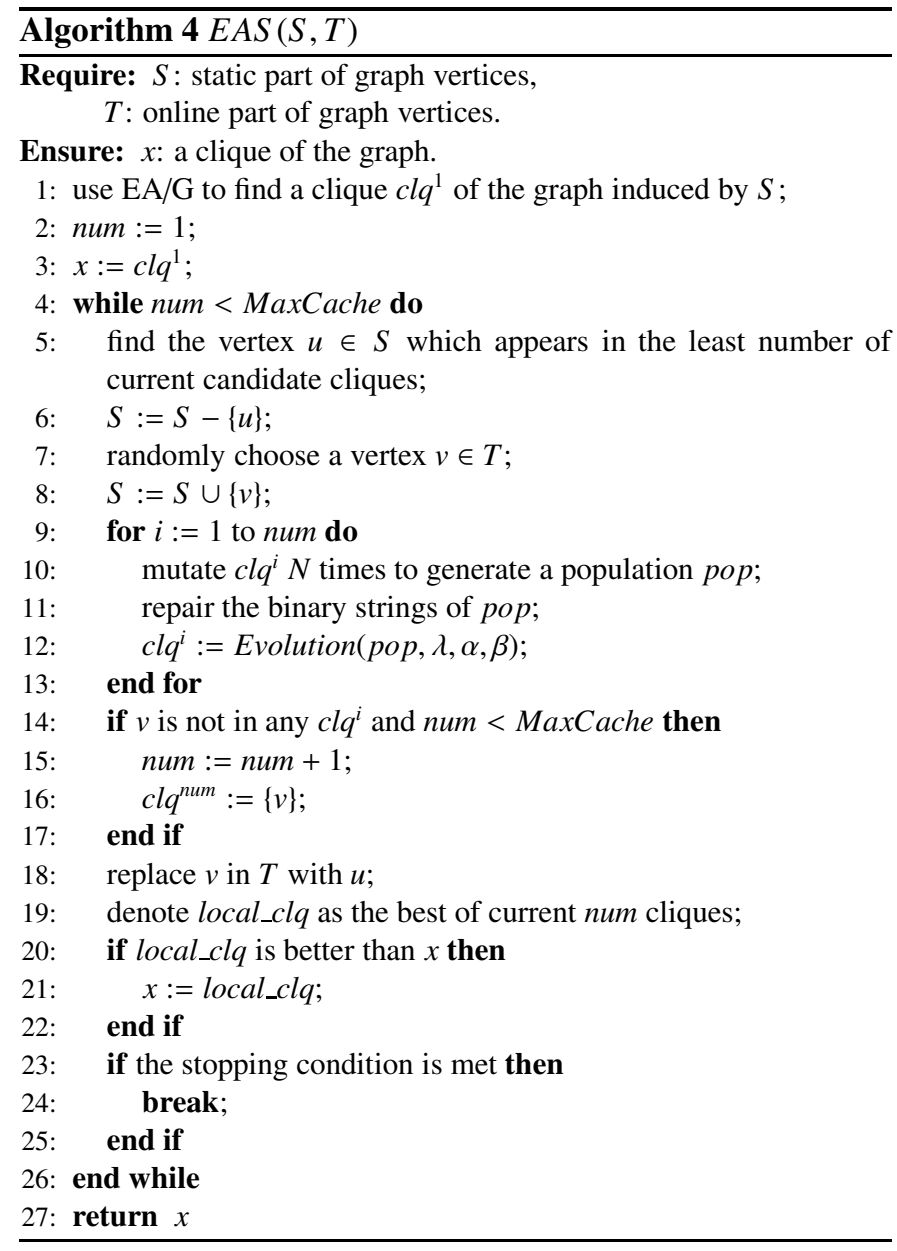

standard EA, such as EA/G. Then, we choose a vertex $u$ from $S$, replace it with a randomly chosen vertex $v$ from $T$, and update the candidate cliques in the pool. In case that $v$ might not be in any of the candidate cliques, we manually create a candidate clique out of $v$. Since the maximum allowed candidates is quite limited (no more than 100), no hash technique is adopted. The algorithm terminates after a given number of substitutions are performed. The pseudo-code of EAS is shown in Algorithm 4.

EAA applies for graphs with a medium size, which can be viewed as a semi-online algorithm. This means that part of the vertices arrive at the same time (the static part $S$ ) and the others arrive one by one (the online part $T$ ). Since the graph cardinality is medium, we can store the whole graph in the memory. Compared with the algorithm EAS, instead of substitution, we can choose a random vertex from $T$ and add it to $S$, and then update the current candidates in the pool.

\subsection{Clique-Based Online Algorithm for Constructing OOCs}

The problem of constructing optimal OOCs can be reduced to the MCP as follows. We first establish a graph in which vertices are codewords that satisfy the auto-correlation property, and edges are added to the pairs of vertices (codewords) that satisfy the cross-correlation property. Then, the maximum OOC can be represented as the maximum clique in the graph.
For the problem of constructing an optimal $\left(v, k, \lambda_{a}, \lambda_{c}\right)$ $\mathrm{OOC}$, the number of vertices in the corresponding graph is usually quite large. In this case, it is difficult for us even to store the graph in memory. Hence, classical MCP algorithms, e.g., $\mathrm{EA} / \mathrm{G}$, may fail to give a meaningful result. In this section, we model the OOC problem as an online version of the MCP with limited memory.

The vertices of the graph are supposed to arrive in a random order, which can be viewed as sampling from the codewords that satisfy the auto-correlation property. Since the computer memory is limited, we restrict the maximum graph cardinality to MaxPoint, and maintain a set of local maximal cliques with the maximum size of the set being MaxCache. If the current graph cardinality is exactly MaxPoint and a new vertex arrives, we must replace one existing vertex with the coming vertex, and then update the set of local optima cliques, like what we do in the EAS algorithm described in Section 3.5.

The whole procedure of the proposed online algorithm for constructing OOCs can be summarized as follows:

Phase 1: Generating a set of non-equivalent codewords

1. Set up an empty codeword set $C$ with size MaxPoint;

2. Generate a binary sequence $c$ with length $v$ and weight $k$ randomly;

3. Convert $c$ into the set-theoretic form and compute its autocorrelation value;

4. If $c$ satisfies the auto-correlation constraint, compute all its multiplier equivalents; otherwise, return to Step 2;

5. Compute the characteristics of the new codewords. If a new codeword is not equivalent to the codewords in $C$, add it to $C$; otherwise, discard it;

6. If the size of $C$ reaches MaxPoint (i.e., the set is full), go to Phase 2; otherwise, return to Step 2.

Phase 2: Building the graph

7. Denote each codeword in the above set $C$ as a vertex of the graph $G$;

8. For each pair of codewords, add an edge to $G$ if the crosscorrelation constraint is satisfied;

9. Generate the MaxPoint $\times$ MaxPoint adjacency matrix of $G$ accordingly.

Phase 3: Finding the maximal clique

10. Denote $S$ as the set of all the MaxPoint vertices of the OOC graph, and $T$ as the set of all other vertices that satisfy the auto-correlation constraint;

11. Find the maximal clique by using the EAS algorithm;

Phase 4: Rewriting the obtained clique as an OOC

12. Expand the characteristics of clique vertices to get the OOC codewords.

In Phase 1, we aim to find all the codewords that satisfy the auto-correlation constraint and put them together to make a codeword set $C$. Then, we convert $C$ into a graph (Phase 2). In Phase 3, we use the EAS algorithm to get a clique: we continue to sample from unchosen graph vertices, replace an existing vertex and update local maximal cliques, until the stopping condition is satisfied (e.g., the maximal number of vertex substitutions is reached). Finally, we rewrite the obtained clique as codewords (Phase 4). 
Table 2: Comparison between EA/G and online algorithms EAA and EAS for the MCP

\begin{tabular}{|c|c|c|c|c|c|c|c|c|c|c|c|c|}
\hline \multirow{2}{*}{ Graph } & \multicolumn{2}{|c|}{ EA/G } & \multicolumn{5}{|c|}{ EAA } & \multicolumn{5}{|c|}{ EAS } \\
\hline & Avg & Best & Avg1 & Avg2 & Best & $p$-value & Time & Avg1 & Avg2 & Best & $p$-value & Time \\
\hline brock200_2 & 12.0 & 12 & 10.2 & 10.7 & 12 & 0.0361 & 0.5 & 10.1 & 11.4 & 12 & $<0.0001$ & 0.9 \\
\hline brock200_4 & 16.5 & 17 & 15.0 & 15.2 & 16 & 0.0165 & 0.5 & 14.9 & 15.7 & 17 & $<0.0001$ & 1.0 \\
\hline brock400_2 & 24.7 & 25 & 23.7 & 23.7 & 24 & 1.0000 & 1.1 & 23.8 & 23.9 & 24 & 0.2201 & 4.9 \\
\hline brock400_4 & 25.1 & 33 & 23.0 & 23.6 & 33 & 0.0957 & 1.1 & 23.0 & 24.1 & 33 & 0.0014 & 4.9 \\
\hline brock800_2 & 20.1 & 21 & 19.1 & 19.2 & 21 & 0.4469 & 3.3 & 19.4 & 19.7 & 20 & 0.0284 & 26.5 \\
\hline brock800_4 & 19.9 & 21 & 19.0 & 19.1 & 21 & 0.5359 & 3.3 & 19.0 & 19.7 & 21 & $<0.0001$ & 26.5 \\
\hline C125.9 & 34.0 & 34 & 30.0 & 31.9 & 33 & $<0.0001$ & 0.4 & 30.0 & 33.8 & 34 & $<0.0001$ & 0.6 \\
\hline C250.9 & 44.0 & 44 & 41.0 & 41.9 & 44 & $<0.0001$ & 0.8 & 41.0 & 43.1 & 44 & $<0.0001$ & 1.8 \\
\hline C500.9 & 55.2 & 56 & 51.8 & 52.4 & 54 & 0.0039 & 1.8 & 51.6 & 53.0 & 55 & $<0.0001$ & 8.7 \\
\hline C1000.9 & 64.4 & 67 & 61.8 & 62.1 & 64 & 0.3045 & 5.8 & 61.9 & 62.6 & 64 & 0.0110 & 51.8 \\
\hline C2000.5 & 14.9 & 16 & 14.6 & 15.0 & 16 & 0.0019 & 24.4 & 14.4 & 15.0 & 16 & $<0.0001$ & 146.2 \\
\hline C2000.9 & 70.9 & 72 & 68.2 & 69.6 & 73 & 0.0004 & 31.0 & 68.1 & 69.4 & 71 & 0.0001 & 318.0 \\
\hline $\mathrm{C} 4000.5$ & 16.1 & 17 & 15.3 & 16.0 & 17 & $<0.0001$ & 137.0 & 15.5 & 16.1 & 17 & $<0.0001$ & 389.3 \\
\hline DSJC500.5 & 13.0 & 13 & 11.8 & 12.1 & 13 & 0.0098 & 1.3 & 12.0 & 12.6 & 13 & $<0.0001$ & 7.1 \\
\hline DSJC1000.5 & 14.5 & 15 & 13.5 & 13.7 & 14 & 0.2264 & 4.6 & 13.2 & 14.0 & 15 & $<0.0001$ & 33.4 \\
\hline gen200_p0.9_44 & 44.0 & 44 & 38.0 & 40.1 & 42 & $<0.0001$ & 0.6 & 38.0 & 41.3 & 44 & $<0.0001$ & 1.2 \\
\hline gen200_p0.9_55 & 55.0 & 55 & 45.0 & 54.0 & 55 & $<0.0001$ & 0.7 & 45.0 & 55.0 & 55 & $<0.0001$ & 1.2 \\
\hline gen400_p0.9_55 & 51.8 & 55 & 46.8 & 48.0 & 50 & $<0.0001$ & 1.3 & 46.8 & 50.0 & 52 & $<0.0001$ & 5.4 \\
\hline gen400_p0.9_65 & 65.0 & 65 & 48.9 & 49.8 & 53 & $<0.0001$ & 1.3 & 48.9 & 53.9 & 64 & $<0.0001$ & 5.3 \\
\hline gen400_p0.9_75 & 75.0 & 75 & 51.2 & 58.8 & 75 & 0.0013 & 1.3 & 50.8 & 72.2 & 75 & $<0.0001$ & 5.4 \\
\hline hamming8-4 & 16.0 & 16 & 13.9 & 16.0 & 16 & $<0.0001$ & 0.6 & 13.9 & 16.0 & 16 & $<0.0001$ & 1.5 \\
\hline hamming 10-4 & 39.8 & 40 & 34.3 & 38.5 & 40 & $<0.0001$ & 5.9 & 34.4 & 39.4 & 40 & $<0.0001$ & 61.8 \\
\hline keller4 & 11.0 & 11 & 11.0 & 11.0 & 11 & - & 0.4 & 11.0 & 11.0 & 11 & - & 0.7 \\
\hline keller5 & 26.9 & 27 & 26.7 & 26.8 & 27 & 0.5925 & 3.2 & 26.4 & 27.0 & 27 & 0.0036 & 29.2 \\
\hline keller6 & 53.4 & 56 & 52.9 & 53.3 & 55 & 0.2154 & 129.5 & 53.4 & 53.6 & 57 & 0.5183 & 558.5 \\
\hline MANN_a27 & 126.0 & 126 & 102.7 & 125.0 & 126 & $<0.0001$ & 2.3 & 102.8 & 124.9 & 125 & $<0.0001$ & 5.1 \\
\hline MANN_a45 & 343.7 & 345 & 275.2 & 337.7 & 342 & $<0.0001$ & 16.9 & 275.6 & 338.6 & 340 & $<0.0001$ & 79.4 \\
\hline MANN_a81 & 1097.2 & 1098 & 870.9 & 929.8 & 938 & $<0.0001$ & 341.9 & 870.7 & 1087.3 & 1089 & $<0.0001$ & 2368.0 \\
\hline p_hat300-1 & 8.0 & 8 & 7.4 & 7.7 & 8 & 0.0086 & 0.6 & 7.4 & 8.0 & 8 & $<0.0001$ & 1.4 \\
\hline p_hat $300-2$ & 25.0 & 25 & 22.0 & 24.3 & 25 & $<0.0001$ & 0.8 & 22.0 & 25.0 & 25 & $<0.0001$ & 2.6 \\
\hline p_hat300-3 & 36.0 & 36 & 30.8 & 33.0 & 34 & $<0.0001$ & 0.8 & 30.8 & 35.6 & 36 & $<0.0001$ & 2.8 \\
\hline p_hat700-1 & 11.0 & 11 & 9.5 & 9.8 & 11 & 0.0621 & 1.9 & 9.5 & 10.8 & 11 & $<0.0001$ & 8.6 \\
\hline p_hat700-2 & 44.0 & 44 & 37.0 & 43.2 & 44 & $<0.0001$ & 2.7 & 37.0 & 43.9 & 44 & $<0.0001$ & 19.9 \\
\hline p_hat700-3 & 62.0 & 62 & 55.0 & 61.1 & 62 & $<0.0001$ & 3.0 & 55.0 & 61.9 & 62 & $<0.0001$ & 26.9 \\
\hline p_hat1500-1 & 11.1 & 12 & 10.3 & 10.8 & 12 & 0.0001 & 9.1 & 10.6 & 11.1 & 12 & 0.0001 & 44.7 \\
\hline p_hat $1500-2$ & 65.0 & 65 & 59.0 & 64.5 & 65 & $<0.0001$ & 16.3 & 59.0 & 64.9 & 65 & $<0.0001$ & 103.9 \\
\hline p_hat $1500-3$ & 93.7 & 94 & 84.6 & 91.3 & 93 & $<0.0001$ & 16.5 & 84.9 & 93.2 & 94 & $<0.0001$ & 160.2 \\
\hline
\end{tabular}

\section{Experimental Study}

In this section, we carry out experiments to verify the performance of proposed algorithms. Two groups of experiments are conducted. The first group of experiments investigates the performance of the proposed EAS and EAA algorithms for solving the MCP, and the second group investigates the performance of the proposed online algorithm for constructing OOCs. All algorithms were implemented in $\mathrm{C}$ and run on an Intel $(3.1 \mathrm{GHz})$ PC with 4GB RAM and Windows 7 operating system.

\subsection{Experimental Results on Solving the MCP}

Since the kernel part of online algorithm for constructing OOCs proposed in this paper is based on EAA and EAS for solving the MCP, this first group of experiments investigates the performance of EAA and EAS, in comparison with EA/G [33], for solving the MCP based on the famous set of 37 DIMACS graphs. The reason we choose EA/G is that our algorithms are built upon EA/G, just with an extra operator of Addition or Substitution under online settings. In this case, we test whether this operator could produce a significant improvement over EA/G for standard benchmarks. Some related parameters of our algorithms were set as follows: $\alpha=0.001$ in the Repair operation, $\beta=0.9$ and $\lambda=0.7$ in the Evolution operation, which are the same as used in [33].

Both EAA and EAS can be divided into two phases: the first phase is to compute a maximal clique using EA/G over $80 \%$ of all the vertices (i.e., the static set $S$ ), and the second phase include the rest $20 \%$ of vertices (i.e., the online set $T$ ). The difference in the second phase between EAS and EAA is that EAA uses an addition operator, while EAS uses a substitution operator, to include the rest $20 \%$ of vertices. In both phases, $M$ was set to be $N / 2$, while $N=10$ for EA/G in the first phase and $N=2$ for the Evolution operation in the second phase.

For each graph, EAA and EAS were run independently for 30 times. The experimental results are shown in Table 2 In Table 2 the second and third columns are the average clique size and the best clique size found by EA/G cited from [33]. The fourth, fifth, sixth and eighth columns are the average maximum clique size found for the $80 \%$ of the vertices by EA/G, the average maximum clique size found by EAA, the overall maximum clique size, and the average execution time in seconds, respec- 
tively. We applied $t$-test to see whether there is any statistical difference between the results achieved before the Addition operation and the results achieved after the Addition operation. The $p$-values are placed in the seventh column.

In Table 2, the 9th to 11th and 13th columns are the average maximum clique size found for the $80 \%$ of the vertices by $\mathrm{EA} / \mathrm{G}$, the average maximum clique size found by EAS, the overall maximum clique size and the average execution time in seconds, respectively. We also apply $t$-test to see statistical differences between the results achieved before the Substitution operation and the results achieved after the Substitution operation. For each run of EAS, the substitution operator was called $n$ times, where $n$ is the number of vertices in the graph. The $p$-values are placed in the 12 th column.

The results show that the average size of the cliques obtained by both EAA and EAS is similar to that of the cliques obtained by EA/G. Both EAA and EAS returned one better clique than EA/G. EAA returns the same best clique size as EA/G does for 22 out of the 37 graphs, while EAS returns the same best clique size as EA/G does for 26 out of the 37 graphs. Both EAA and EAS achieve a clique with the best size only one smaller than that achieved by EA/G on 6 graphs, respectively.

It can be seen that we did not obtain better results than EA/G over most of the DIMACS graphs, especially for dense graphs. The main reason is that EA/G requires full information of the input graph and performs a more thorough search (the Repair operator was called 2000 times), while EAA and EAS only perform a limited search (two individuals would converge soon) over each updated graph. Even so, the difference among the results from EA/G, EAA and EAS is quite small. It should also be pointed out that the $t$-test shows a promising result that both of Addition and Substitution operators improve local optimal cliques significantly.

In the following subsections, we will first show the difficulty of searching OOCs by computer algorithms in Section 4.2 and then investigate the performance of our online algorithm to construct OOCs in Section 4.3. Compared with DIMACS graphs (where the graph size is less than 5000), OOC graphs have much bigger sizes (often bigger than 10,000) and sparser structures. Hence, we just test EAS to search for OOCs.

\subsection{Experimental Results on Generating OOC Graphs}

To show the difficulty of searching OOCs by computer algorithms, we present the graph cardinalities for $(v, 4,2)-,(v, 5,2)$ and $(v, 6,2)$-OOCs. Since no mathematical formula can be found, we use computer algorithm to get a lower bound. As introduced in the previous section, an isomorphism technique can be used to accelerate this procedure. Firstly, we iteratively generate a codeword that satisfies the code length and code weight conditions at random until we get a codeword that satisfies the OOC auto-correlation constraint. Then, we use the isomorphism mapping to get more OOC codewords. The procedure continues until we have performed 100,000 random searches. The results are presented in Fig. 1 and Table 3 .

It can be seen that the graph size increases quickly with the growth of the OOC code length. Taking the $(300,4,2)$-OOC for

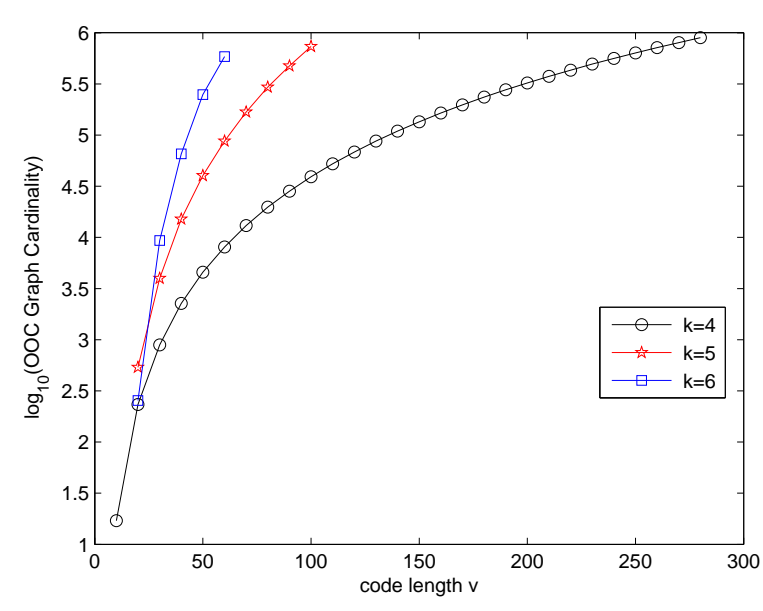

Figure 1: Fast increasing of the graph cardinality.

instance, the corresponding graph size is already over one million, and just storing such a graph in memory is difficult. In this case, traditional off-line algorithm may fail to obtain good results. In order to overcome the limitation of computer memory for storing large graphs, part of the graph vertices are supposed to arrive sequentially to be fed into the algorithm, and an update operation is performed to improve current candidate cliques.

\subsection{Experimental Results on Constructing OOCs}

In this group of experiments, we investigate our proposed online algorithm based on EAS to get $\left(v, k, \lambda_{a}, \lambda_{c}\right)$-OOCs with some specific parameters. Parameters $\alpha, \beta$ and $\lambda$ were set to be the same as used in the first group of experiments. In the first phase of EAS, the population size $N$ was set to be 10 for EA/G, $M=N / 2=5$, and the Repair operation within EA/G was run 20,000 times, as we did for DIMACS graphs. In the second phase of EAS, the population size $N$ was set to 2 for the Evolution operation, $M=N / 2=1$, and the algorithm was terminated after 2,000 vertex substitutions. The number of candidate cliques, MaxCache, was set to be 100, and the parameter MaxPoint was set to 10,000 .

Here, we give an explanation for above settings. Since our computers just have 4GB RAM, and the graph is stored as adjacency matrices, then the graph size should be less than 100,000. But, if the graph size is too small, e.g., 5000, the optimal OOC size will be small, and we can not observe significant differences. Therefore, we set MaxPoint to be 10,000.

During the Substitution phase of EAS, the population size $N$ was set to 2 , and the number of candidate cliques was set to 100. These parameters were set to be small for the reason of running times. Taking the $(300,4,2)$-OOC for example, even under this setting of small parameters, it still takes about three hours to perform a single run.

For decades, different researchers have devoted effort to the research of OOCs, many good results have been obtained by an extensive use of various mathematical tools. Most of these results work for code weight $k=4$ or $k=5$, and correlation constraint no more than 2 . For bigger code weight or correlation constraint, hardly any theoretical result is known. Therefore 
Table 3: Lower bound of $(v, k, 2)$-OOC Graphs

\begin{tabular}{cc|cc|cc|cc|cc}
\hline$(v, k)$ & Cardinality & $(v, k)$ & Cardinality & $(v, k)$ & Cardinality & $(v, k)$ & Cardinality & $(v, k)$ & Cardinality \\
\hline$(10,4)$ & 17 & $(100,4)$ & 39151 & $(190,4)$ & 276686 & $(280,4)$ & 894813 & $(90,5)$ & 474370 \\
$(20,4)$ & 233 & $(110,4)$ & 52411 & $(200,4)$ & 323251 & $(290,4)$ & $>1000000$ & $(100,5)$ & 735269 \\
$(30,4)$ & 889 & $(120,4)$ & 68328 & $(210,4)$ & 374705 & $(20,5)$ & 538 & $(110,5)$ & $>1000000$ \\
$(40,4)$ & 2263 & $(130,4)$ & 87297 & $(220,4)$ & 431418 & $(30,5)$ & 3984 & $(20,6)$ & 255 \\
$(50,4)$ & 4577 & $(140,4)$ & 109404 & $(230,4)$ & 493627 & $(40,5)$ & 15118 & $(30,6)$ & 9342 \\
$(60,4)$ & 8075 & $(150,4)$ & 134917 & $(240,4)$ & 561385 & $(50,5)$ & 40120 & $(40,6)$ & 65564 \\
$(70,4)$ & 13057 & $(160,4)$ & 164121 & $(250,4)$ & 635290 & $(60,5)$ & 87258 & $(50,6)$ & 248510 \\
$(80,4)$ & 19723 & $(170,4)$ & 197447 & $(260,4)$ & 715174 & $(70,5)$ & 168177 & $(60,6)$ & 586090 \\
$(90,4)$ & 28303 & $(180,4)$ & 234773 & $(270,4)$ & 801532 & $(80,5)$ & 293850 & $(70,6)$ & $>1000000$ \\
\hline
\end{tabular}

the proposed algorithm is tested to design $(v, 4,2)-,(v, 5,2)$ and $(v, 4,2,1)$-OOCs, where the existence results are rich, and also indicating that the proposed algorithm can be used under different settings of code weights and correlation constraints.

\subsubsection{Constructing $(v, 4,2)-O O C s$}

Since the construction on $(v, 4,2)$-OOCs with small orders was thoroughly discussed in [14, 18], we first test our algorithm EAS for $(v, 4,2)$-OOCs with $10 \leq v \leq 64$ (with the graph size less than 10,000), each with 30 runs. As pointed in Section 2, Chu's branch-and-bound algorithm [14] searches for optimal solutions and can not be generalized to an arbitrary setting of parameters, we compare EAS with its source algorithm EA/G.

The results are shown in Table 4, where the first four columns are the code length $(v)$, correspondent graph cardinality (Cardinality), Johnson bound (Bound), and existing result cited from Feng [18] (Existing) (where "??" means that there is no existing result for the corresponding case), respectively. The following six columns are the average code size ( $A v g)$, best code size (Best), and average running time (Time) returned by EA/G and EAS, respectively. The last column shows the $p$ value of applying the $t$-test to compare EAS over EA/G. In the table, for each setting of $v$, if EAS significantly outperforms $\mathrm{EA} / \mathrm{G}$, its best code size is bold-faced.

From Table 4, it is not hard to see that EAS has a better result than EA/G for all the cases when $v \leq 64$. Especially, when $v \geq 32$, the difference is significant $(p<0.0001)$. For the case of $v>64$, the corresponding OOC graph has more than 10,000 vertices, and hence traditional off-line algorithm, such as EA/G, may fail to give a result due to the computer memory limitation in our settings.

Here, we also test EA/G for randomly generated OOC graphs when $v>64$ (with the graph size 10,000), while EAS would do another 2000 vertex substitutions. Both EA/G and EAS were run 30 times for each code length. The results are listed in Table 5, where for each setting of $v$ if EAS significantly outperforms EA/G, its best code size is bold-faced. It can been seen that the substitution operation improves current solutions significantly, indicating that EAS outperforms EA/G when constructing $(v, 4,2)$-OOCs.

\subsubsection{Constructing $(v, 5,2)-O O C s$}

For the case of OOCs with a bigger weight, there is hardly any result on the existence of maximum codes [3]. However, we can still obtain some computing results in Table 6 for $v \leq 100$.
From Table 6, it can be seen that EAS performs better for $(v, 4,2)$ than for $(v, 5,2)$. This is because, with the growth of the values of $v$ and $k$, the corresponding graph may become huge and sparse, and hence, the searching for a feasible clique is more difficult in nature.

It should be pointed out that the obtained best code sizes for $(300,4,2)$-OOC and $(100,5,2)-O O C$ both reach $63 \%$ of their Johnson bounds, respectively. Figs. 2 (a) and (b) show the percentage of the obtained best code size to the corresponding Johnson bound and the percentage of the number of stored nodes to the number of nodes in the OOC graph (i.e., the cardinality of the OOC graph) against the code length on $(v, 4,2)$ OOC and $(v, 5,2)-O O C$, respectively. Note that in Fig.2(a), the values of $y$ for $v=290$ and $v=300$ are not plotted because their corresponding graph cardinalities are over one million, in which cases we just know that the percentages are less than $1 \%$, but they can not be calculated precisely.

From Fig. 2, it can be seen that both the percentages drop with the growing of the code length. Actually, we just stored less than $1 \%$ of the OOC graph nodes, but obtained $63 \%$ of the Johnson bound regarding the OOC code size. Moreover, the Johnson bound is a theoretical upper bound, whether it can be achieved or not is still unknown for many parameters. If we are admitted more computer memory and more running time, the result would be surely improved.

\subsubsection{Constructing $(v, 4,2,1)$-OOCs}

For the case that auto- and cross-correlation constraints are unequal, we choose to construct $(v, 4,2,1)$-OOCs for $10 \leq v \leq$ 100. For $(v, 4,2,1)$-OOCs, Buratti [12] has proved the following bound:

$$
s \leq \begin{cases}\left\lceil\frac{v}{8}\right\rceil, & \text { for } v \equiv 7,14 \quad(\bmod 56) \\ \left\lfloor\frac{v}{8}\right\rfloor, & \text { otherwise }\end{cases}
$$

The experimental results are shown in Table 7, where the bound information is calculated from Eq. (15) directly. Compared with our result, Baicheva and Topalova [4] ran a parallel backtrack search algorithm on IBM supercomputers to find the optimal $(v, 4,2,1)$-OOCs for $v \leq 181$. However, their algorithm is equivalent to clique search: with the growth of code length, the corresponding graph structure can not be stored in even supercomputers, thus their off-line backtracking can not be applied for general bigger $v$. Note that we are aiming to get OOCs of relatively large sizes for general parameters. In this sense, EAS ourperforms backtracking clearly. 
Table 4: Results on constructing $(v, 4,2)$-OOCs for $10 \leq v \leq 64$

\begin{tabular}{|c|c|c|c|c|c|c|c|c|c|c|}
\hline \multirow{2}{*}{$v$} & \multirow{2}{*}{ Cardinality } & \multirow{2}{*}{ Bound } & \multirow{2}{*}{ Existing } & \multicolumn{3}{|c|}{$\overline{\mathrm{EA} / \mathrm{G}}$} & \multicolumn{4}{|c|}{ EAS } \\
\hline & & & & Avg & Best & Time & Avg & Best & Time & $p$-value \\
\hline 10 & 17 & 3 & Yes & 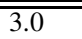 & $\overline{\bar{z}}$ & $\overline{0.2}$ & 2.6 & $\overline{\overline{3}}$ & $\overline{0.2}$ & 0.0001 \\
\hline 11 & 25 & 3 & Yes & 3.0 & 3 & 0.2 & 3.0 & 3 & 0.2 & - \\
\hline 12 & 34 & 4 & No & 3.0 & 3 & 0.2 & 3.0 & 3 & 0.2 & - \\
\hline 13 & 49 & 5 & No & 4.0 & 4 & 0.3 & 4.0 & 4 & 0.3 & - \\
\hline 14 & 64 & 6 & Yes & 5.9 & 6 & 0.3 & 5.5 & 6 & 0.3 & 0.0033 \\
\hline 15 & 82 & 7 & Yes & 6.8 & 7 & 0.4 & 6.2 & 7 & 0.3 & $<0.0001$ \\
\hline 16 & 106 & 8 & Yes & 8.0 & 8 & 0.5 & 7.7 & 8 & 0.4 & 0.0213 \\
\hline 17 & 132 & 9 & Yes & 9.0 & 9 & 0.6 & 9.0 & 9 & 0.5 & - \\
\hline 18 & 156 & 11 & Yes & 10.2 & 11 & 0.6 & 10.3 & 11 & 0.5 & 0.7558 \\
\hline 19 & 195 & 12 & Yes & 11.7 & 12 & 0.8 & 11.2 & 12 & 0.8 & 0.0002 \\
\hline 20 & 233 & 14 & Yes & 13.2 & 14 & 0.9 & 13.0 & 14 & 0.8 & 0.0815 \\
\hline 21 & 270 & 15 & Yes & 14.1 & 15 & 1.0 & 14.1 & 15 & 0.9 & 0.6921 \\
\hline 22 & 320 & 17 & Yes & 16.2 & 17 & 1.1 & 16.1 & 17 & 1.1 & 0.7212 \\
\hline 23 & 374 & 18 & Yes & 17.7 & 18 & 1.5 & 17.5 & 18 & 1.4 & 0.1001 \\
\hline 24 & 424 & 21 & No & 19.2 & 20 & 1.5 & 19.1 & 20 & 1.5 & 0.2320 \\
\hline 25 & 495 & 22 & Yes & 21.0 & 21 & 1.9 & 21.0 & 21 & 2.1 & 0.3219 \\
\hline 26 & 560 & 25 & Yes & 22.9 & 23 & 2.0 & 23.1 & 24 & 2.3 & 0.0250 \\
\hline 27 & 630 & 26 & Yes & 24.5 & 25 & 2.4 & 24.5 & 25 & 2.9 & 1.0000 \\
\hline 28 & 716 & 29 & Yes & 26.4 & 27 & 2.6 & 26.4 & 27 & 3.4 & 1.0000 \\
\hline 29 & 805 & 30 & Yes & 28.4 & 29 & 3.3 & 28.8 & 30 & 4.2 & 0.0037 \\
\hline 30 & 889 & 33 & Yes & 30.4 & 31 & 3.2 & 30.9 & 32 & 4.5 & 0.0001 \\
\hline 31 & 1000 & 35 & Yes & 32.5 & 33 & 4.1 & 33.0 & 34 & 6.1 & 0.0002 \\
\hline 32 & 1106 & 38 & Yes & 34.7 & 36 & 4.5 & 35.4 & 36 & 6.8 & $<0.0001$ \\
\hline 33 & 1215 & 40 & Yes & 37.0 & 38 & 4.9 & 37.6 & 38 & 8.0 & $<0.0001$ \\
\hline 34 & 1344 & 44 & Yes & 39.2 & 40 & 5.5 & 40.1 & 41 & 9.6 & $<0.0001$ \\
\hline 35 & 1480 & 45 & Yes & 41.7 & 43 & 6.7 & 42.7 & 44 & 11.5 & $<0.0001$ \\
\hline 36 & 1606 & 49 & Yes & 44.1 & 45 & 7.0 & 45.1 & 46 & 12.8 & $<0.0001$ \\
\hline 37 & 1767 & 51 & Yes & 46.3 & 47 & 8.5 & 48.0 & 49 & 15.6 & $<0.0001$ \\
\hline 38 & 1920 & 55 & Yes & 49.2 & 50 & 9.0 & 50.6 & 51 & 18.2 & $<0.0001$ \\
\hline 39 & 2079 & 57 & Yes & 51.9 & 53 & 11.2 & 53.3 & 55 & 21.3 & $<0.0001$ \\
\hline 40 & 2263 & 61 & Yes & 54.5 & 55 & 13.6 & 56.4 & 58 & 24.4 & $<0.0001$ \\
\hline 41 & 2450 & 63 & Yes & 57.4 & 59 & 15.7 & 59.5 & 60 & 28.5 & $<0.0001$ \\
\hline 42 & 2628 & 68 & Yes & 60.3 & 62 & 17.0 & 62.7 & 63 & 31.8 & $<0.0001$ \\
\hline 43 & 2849 & 70 & Yes & 63.4 & 64 & 20.9 & 65.8 & 67 & 41.3 & $<0.0001$ \\
\hline 44 & 3060 & 75 & Yes & 66.4 & 67 & 22.8 & 69.0 & 70 & 47.6 & $<0.0001$ \\
\hline 45 & 3277 & 77 & $? ?$ & 69.2 & 70 & 26.4 & 71.9 & 73 & 54.9 & $<0.0001$ \\
\hline 46 & 3520 & 82 & Yes & 72.7 & 74 & 29.1 & 75.5 & 77 & 62.8 & $<0.0001$ \\
\hline 47 & 3772 & 84 & $? ?$ & 75.9 & 77 & 34.1 & 79.1 & 80 & 71.2 & $<0.0001$ \\
\hline 48 & 4012 & 90 & No & 79.3 & 81 & 36.8 & 82.4 & 84 & 79.7 & $<0.0001$ \\
\hline 49 & 4300 & 92 & Yes & 82.4 & 84 & 42.6 & 86.0 & 87 & 91.3 & $<0.0001$ \\
\hline 50 & 4577 & 98 & Yes & 86.2 & 88 & 45.6 & 89.7 & 92 & 98.3 & $<0.0001$ \\
\hline 51 & 4860 & 100 & Yes & 89.9 & 91 & 51.2 & 93.6 & 95 & 111.3 & $<0.0001$ \\
\hline 52 & 5176 & 106 & Yes & 93.3 & 95 & 56.4 & 97.3 & 98 & 126.1 & $<0.0001$ \\
\hline 53 & 5499 & 108 & $? ?$ & 97.1 & 98 & 64.2 & 101.1 & 102 & 138.0 & $<0.0001$ \\
\hline 54 & 5808 & 114 & Yes & 100.6 & 102 & 67.7 & 105.0 & 106 & 151.9 & $<0.0001$ \\
\hline 55 & 6175 & 117 & $? ?$ & 104.5 & 105 & 76.9 & 109.1 & 111 & 168.6 & $<0.0001$ \\
\hline 56 & 6526 & 123 & Yes & 108.7 & 110 & 84.5 & 113.2 & 114 & 181.9 & $<0.0001$ \\
\hline 57 & 6885 & 126 & Yes & 112.7 & 114 & 92.7 & 117.8 & 119 & 197.2 & $<0.0001$ \\
\hline 58 & 7280 & 133 & Yes & 116.6 & 118 & 100.9 & 122.1 & 124 & 213.2 & $<0.0001$ \\
\hline 59 & 7685 & 135 & $? ?$ & 121.0 & 122 & 113.7 & 126.3 & 128 & 238.1 & $<0.0001$ \\
\hline 60 & 8075 & 142 & $? ?$ & 124.9 & 126 & 121.7 & 130.7 & 133 & 254.8 & $<0.0001$ \\
\hline 61 & 8525 & 145 & Yes & 129.5 & 130 & 137.2 & 135.2 & 137 & 274.7 & $<0.0001$ \\
\hline 62 & 8960 & 152 & Yes & 133.8 & 136 & 146.3 & 139.7 & 141 & 295.9 & $<0.0001$ \\
\hline 63 & 9405 & 155 & Yes & 138.2 & 139 & 163.4 & 144.5 & 147 & 331.1 & $<0.0001$ \\
\hline 64 & 9882 & 162 & Yes & 142.8 & 144 & 182.0 & 149.0 & 150 & 320.2 & $<0.0001$ \\
\hline
\end{tabular}

\section{Conclusions and Future Work}

Due to its great importance in the field of communications, the problem of designing OOCs has attracted increasing attentions in recent years. Many good results have been obtained by an extensive use of various mathematical tools. But, it seems that we can not expect the problem to be completely solved for arbitrary parameters in the near future. Meanwhile, the design of OOCs can be reduced to the maximum clique problem (MCP) in graph theory, for which there are many successful heuristics. As a result, we propose clique-based heuristics to design OOCs with relatively large sizes.

In this paper, we present an online algorithm for constructing OOCs based on an EA with guided mutation $(\mathrm{EA} / \mathrm{G})[33]$. 
Table 5: Results on constructing $(v, 4,2)$-OOCs for bigger $v$

\begin{tabular}{cccc||c|c|c|c|c|l|l}
\hline \multirow{2}{*}{$v$} & \multirow{2}{*}{ Cardinality } & \multirow{2}{*}{ Bound } & \multirow{2}{*}{ Existing } & \multicolumn{3}{c|}{ EA/G } & \multicolumn{3}{|c}{ EAS } \\
\cline { 5 - 9 } & & & Avg & Best & Time & Avg & Best & Time & $p$-value \\
\hline \hline 70 & 13057 & 195 & Yes & 169.0 & 170 & 189.0 & 179.3 & $\mathbf{1 8 1}$ & 689.0 & $<0.0001$ \\
80 & 19723 & 256 & Yes & 216.3 & 220 & 223.8 & 233.3 & $\mathbf{2 3 6}$ & 2722.5 & $<0.0001$ \\
90 & 28303 & 326 & Yes & 269.8 & 274 & 258.1 & 293.1 & $\mathbf{2 9 4}$ & 4631.9 & $<0.0001$ \\
100 & 39151 & 404 & Yes & 327.3 & 330 & 286.6 & 357.4 & $\mathbf{3 5 9}$ & 6295.3 & $<0.0001$ \\
110 & 52411 & 490 & Yes & 392.4 & 394 & 328.1 & 428.2 & $\mathbf{4 2 9}$ & 7673.7 & $<0.0001$ \\
120 & 68328 & 585 & No & 472.8 & 480 & 358.8 & 502.8 & $\mathbf{5 0 4}$ & 7967.5 & $<0.0001$ \\
130 & 87297 & 688 & Yes & 569.3 & 577 & 391.3 & 588.5 & $\mathbf{5 9 4}$ & 8976.4 & $<0.0001$ \\
140 & 109404 & 799 & Yes & 663.5 & 666 & 419.4 & 679.0 & $\mathbf{6 8 0}$ & 9389.4 & $<0.0001$ \\
150 & 134917 & 918 & Yes & 757.3 & 759 & 452.3 & 775.5 & $\mathbf{7 8 2}$ & 9654.4 & $<0.0001$ \\
160 & 164121 & 1046 & Yes & 840.8 & 862 & 479.1 & 865.3 & $\mathbf{8 8 3}$ & 9934.2 & $<0.0001$ \\
\hline 170 & 197447 & 1183 & Yes & 961.3 & 973 & 521.9 & 981.0 & $\mathbf{9 8 9}$ & 9989.8 & $<0.0001$ \\
180 & 234773 & 1327 & Yes & 1009.0 & 1033 & 546.5 & 1041.0 & $\mathbf{1 0 6 2}$ & 10204.1 & $<0.0001$ \\
190 & 276686 & 1480 & Yes & 1177.5 & 1188 & 593.5 & 1204.5 & $\mathbf{1 2 1 8}$ & 10143.6 & $<0.0001$ \\
200 & 323251 & 1641 & Yes & 1237.8 & 1250 & 616.3 & 1270.3 & $\mathbf{1 2 7 7}$ & 9876.0 & $<0.0001$ \\
210 & 374705 & 1811 & Yes & 1351.0 & 1361 & 662.1 & 1390.5 & $\mathbf{1 3 9 6}$ & 10069.0 & $<0.0001$ \\
220 & 431418 & 1989 & Yes & 1421.3 & 1458 & 694.6 & 1472.8 & $\mathbf{1 5 0 6}$ & 9846.9 & $<0.0001$ \\
230 & 493627 & 2175 & Yes & 1630.3 & 1684 & 761.7 & 1673.0 & $\mathbf{1 7 2 0}$ & 9667.3 & $<0.0001$ \\
240 & 561385 & 2370 & No & 1538.3 & 1556 & 743.8 & 1622.3 & $\mathbf{1 6 4 7}$ & 8717.4 & $<0.0001$ \\
250 & 635290 & 2573 & Yes & 1825.3 & 1860 & 832.3 & 1882.3 & $\mathbf{1 9 0 4}$ & 9776.8 & $<0.0001$ \\
260 & 715174 & 2784 & Yes & 1845.5 & 1930 & 853.9 & 1929.3 & $\mathbf{2 0 0 6}$ & 9187.4 & $<0.0001$ \\
\hline 270 & 801532 & 3003 & Yes & 1962.8 & 1990 & 903.4 & 2044.0 & $\mathbf{2 0 6 1}$ & 9303.7 & $<0.0001$ \\
280 & 894813 & 3231 & Yes & 1925.5 & 2005 & 905.8 & 2042.8 & $\mathbf{2 1 1 0}$ & 9392.4 & $<0.0001$ \\
290 & $>1000000$ & 3468 & Yes & 2293.5 & 2319 & 1025.2 & 2382.0 & $\mathbf{2 4 0 4}$ & 8656.9 & $<0.0001$ \\
300 & $>1000000$ & 3712 & $? ?$ & 2108.7 & 2211 & 1041.9 & 2255.7 & $\mathbf{2 3 4 7}$ & 7937.8 & $<0.0001$ \\
\hline
\end{tabular}

Table 6: Results on constructing $(v, 5,2)$-OOCs for $10 \leq v \leq 100$

\begin{tabular}{|c|c|c|c|c|c|c|c|c|c|c|c|c|c|c|}
\hline$v$ & Bound & EAS & $v$ & Bound & EAS & $v$ & Bound & EAS & $v$ & Bound & EAS & $v$ & Bound & EAS \\
\hline 10 & 0 & 0 & 29 & 12 & 9 & 48 & 35 & 26 & 67 & 69 & 49 & 86 & 119 & 76 \\
\hline 11 & 1 & 0 & 30 & 13 & 10 & 49 & 36 & 27 & 68 & 73 & 49 & 87 & 120 & 78 \\
\hline 12 & 1 & 1 & 31 & 13 & 11 & 50 & 39 & 28 & 69 & 74 & 51 & 88 & 121 & 81 \\
\hline 13 & 1 & 1 & 32 & 15 & 12 & 51 & 40 & 29 & 70 & 75 & 52 & 89 & 127 & 81 \\
\hline 14 & 2 & 1 & 33 & 16 & 12 & 52 & 40 & 30 & 71 & 80 & 54 & 90 & 129 & 83 \\
\hline 15 & 2 & 2 & 34 & 16 & 13 & 53 & 44 & 31 & 72 & 81 & 55 & 91 & 130 & 84 \\
\hline 16 & 3 & 2 & 35 & 18 & 14 & 54 & 45 & 32 & 73 & 82 & 56 & 92 & 136 & 88 \\
\hline 17 & 4 & 4 & 36 & 19 & 15 & 55 & 45 & 34 & 74 & 87 & 58 & 93 & 138 & 88 \\
\hline 18 & 4 & 3 & 37 & 19 & 15 & 56 & 49 & 35 & 75 & 88 & 60 & 94 & 139 & 90 \\
\hline 19 & 4 & 4 & 38 & 22 & 17 & 57 & 50 & 36 & 76 & 90 & 61 & 95 & 145 & 92 \\
\hline 20 & 5 & 4 & 39 & 22 & 18 & 58 & 51 & 37 & 77 & 95 & 64 & 96 & 147 & 93 \\
\hline 21 & 6 & 5 & 40 & 23 & 18 & 59 & 55 & 38 & 78 & 96 & 63 & 97 & 148 & 94 \\
\hline 22 & 6 & 5 & 41 & 26 & 19 & 60 & 56 & 39 & 79 & 97 & 65 & 98 & 155 & 97 \\
\hline 23 & 7 & 6 & 42 & 26 & 20 & 61 & 57 & 40 & 80 & 102 & 67 & 99 & 156 & 100 \\
\hline 24 & 8 & 6 & 43 & 27 & 21 & 62 & 61 & 43 & 81 & 104 & 69 & 100 & 158 & 100 \\
\hline 25 & 8 & 7 & 44 & 30 & 22 & 63 & 62 & 43 & 82 & 105 & 71 & & & \\
\hline 26 & 10 & 7 & 45 & 30 & 23 & 64 & 63 & 44 & 83 & 110 & 72 & & & \\
\hline 27 & 10 & 8 & 46 & 31 & 24 & 65 & 67 & 46 & 84 & 112 & 73 & & & \\
\hline 28 & 10 & 9 & 47 & 34 & 25 & 66 & 68 & 47 & 85 & 113 & 75 & & & \\
\hline
\end{tabular}

Note that the cardinality of the OOC graph is usually huge and the corresponding clique size is quite limited. Hence, the usual clique-based strategy may fail in this case. So, we suppose all the graph vertices arrive in an online manner, and present a substitution technique which leads to our EA with substitution (EAS). Experiments show that EAS has a similarly good performance to EA/G for the MCP on DIMACS benchmarks. Then, we use EAS to construct OOCs with different code weights and different correlation constraints. The experimental study shows that EAS returns OOCs of sizes close to the current best upper bound when the code length is small. Taking $(v, 4,2)$ for instance, the ratio of the obtained code size to the Johnson bound is over $90 \%$ when $v<100$. If more running time is admitted, the result could be improved.

It should be pointed out that our algorithm is based on EA/G. The main reason for such a choice is its balance between simplicity and efficiency. In fact, there are some existing clique algorithms which have better performance [8, 10]. This would be a subject for the future work.

\section{Acknowledgments}

The work was supported by the National Natural Science Foundation of China (NSFC) under Grant 11401317 and Grant 


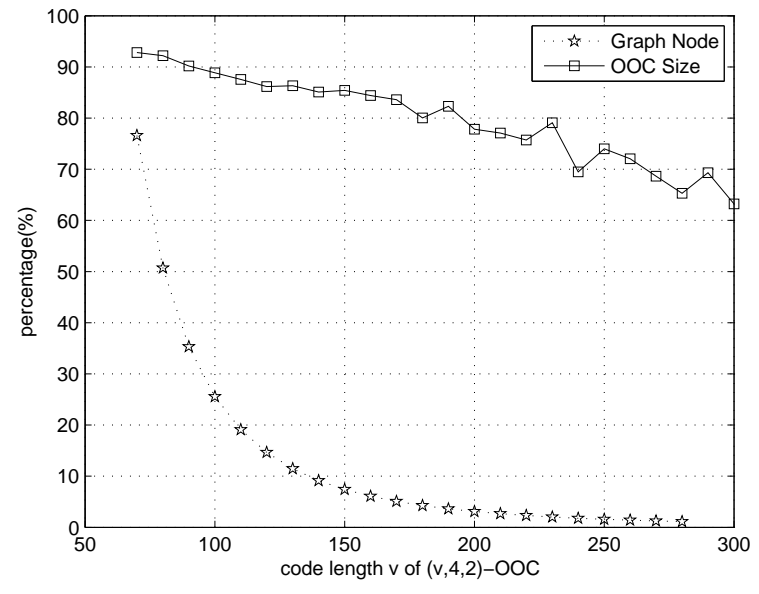

(a)

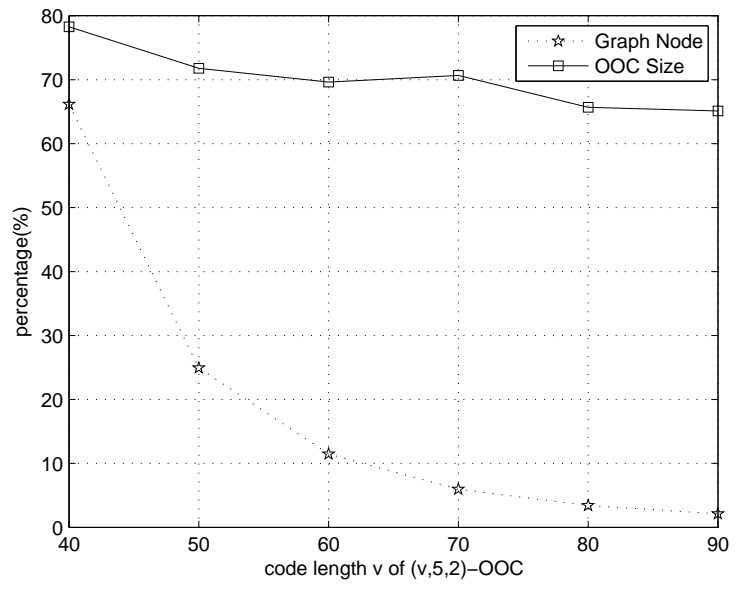

(b)

Figure 2: The percentage of the obtained best code size to the Johnson bound and percentage of the number of stored nodes to the number of nodes in the OOC graph against the code length: (a) $(v, 4,2)-\mathrm{OOC}$ and (b) $(v, 5,2)-\mathrm{OOC}$.

Table 7: Results on constructing $(v, 4,2,1)$-OOCs for $10 \leq v \leq 100$

\begin{tabular}{ccc|ccc|ccc|cccccc}
\hline$v$ & Bound & EAS & $v$ & Bound & EAS & $v$ & Bound & EAS & $v$ & Bound & EAS & $v$ & Bound & EAS \\
\hline 10 & 1 & 1 & 29 & 3 & 3 & 48 & 6 & 5 & 67 & 8 & 8 & 86 & 10 & 10 \\
11 & 1 & 1 & 30 & 3 & 3 & 49 & 6 & 6 & 68 & 8 & 8 & 87 & 10 & 10 \\
12 & 1 & 1 & 31 & 3 & 3 & 50 & 6 & 6 & 69 & 8 & 8 & 88 & 11 & 10 \\
13 & 1 & 1 & 32 & 4 & 3 & 51 & 6 & 5 & 70 & 9 & 8 & 89 & 11 & 10 \\
14 & 2 & 1 & 33 & 4 & 3 & 52 & 6 & 6 & 71 & 8 & 8 & 90 & 11 & 10 \\
15 & 1 & 1 & 34 & 4 & 4 & 53 & 6 & 6 & 72 & 9 & 8 & 91 & 11 \\
16 & 2 & 1 & 35 & 4 & 4 & 54 & 6 & 6 & 73 & 9 & 8 & 92 & 11 \\
17 & 2 & 2 & 36 & 4 & 4 & 55 & 6 & 6 & 74 & 9 & 9 & 93 & 11 \\
18 & 2 & 1 & 37 & 4 & 4 & 56 & 7 & 7 & 75 & 9 & 9 & 94 & 11 \\
19 & 2 & 2 & 38 & 4 & 4 & 57 & 7 & 6 & 76 & 9 & 8 & 95 & 11 \\
20 & 2 & 2 & 39 & 4 & 4 & 58 & 7 & 7 & 77 & 9 & 9 & 96 & 11 \\
21 & 2 & 2 & 40 & 5 & 5 & 59 & 7 & 7 & 78 & 9 & 9 & 97 & 11 \\
22 & 2 & 2 & 41 & 5 & 5 & 60 & 7 & 7 & 79 & 9 & 9 & 98 & 12 \\
23 & 2 & 2 & 42 & 5 & 5 & 61 & 7 & 7 & 80 & 10 & 9 & 99 & 11 \\
24 & 3 & 2 & 43 & 5 & 5 & 62 & 7 & 7 & 81 & 10 & 9 & 11 \\
25 & 3 & 3 & 44 & 5 & 5 & 63 & 8 & 7 & 82 & 10 & 10 & 11 \\
26 & 3 & 3 & 45 & 5 & 5 & 64 & 8 & 7 & 83 & 10 & 10 & 11 \\
27 & 3 & 2 & 46 & 5 & 5 & 65 & 8 & 8 & 84 & 10 & 9 & 11 \\
28 & 3 & 3 & 47 & 5 & 5 & 66 & 8 & 8 & 85 & 10 & 9 & \\
\hline
\end{tabular}

11126291, Nanjing University of Information Science and Technology under Grant 2012x021, and the Engineering and Physical Sciences Research Council (EPSRC) of U. K. under Grant EP/K001310/1. The authors would like to thank the anonymous reviewers for their valuable comments.

\section{References}

[1] R. J. R. Abel, M. Buratti, Some progress on (v, 4, 1) difference families and optical orthogonal codes, J. Combin. Theory Ser. A 106 (1) (2004) 59-75.

[2] T. Alderson, K. Mellinger, Geometric constructions of optimal optical orthogonal codes, Adv. Math. Commun 2 (4) (2008) 451-467.

[3] T. Alderson, K. Mellinger, Families of optimal oocs with $\lambda=2$, IEEE Trans. Inf. Theory 54 (8) (2008) 3722-3724.

[4] T. Baicheva, S. Topalova, Optimal $(v, 4,2,1)$ optical orthogonal codes with small parameters, J. Combin. Des. 20 (2) (2012) 142-160.

[5] T. Baicheva, S. Topalova, Optimal (v, 5, 2, 1) optical orthogonal codes of small v, Applicable Algebra in Engineering, Communication and Computing 24 (2013) 165-177.
[6] S. Baluja, Population-based incremental learning: A method for integrating genetic search based function optimization and competitive learning, Tech. rep., CMU-CS-94-163, Carnegie Mellon University (1994).

[7] R. Battiti, F. Mascia, Reactive and dynamic local search for max-clique: Engineering effective building blocks, Comput. Oper. Res. 37 (3) (2010) 534-542.

[8] R. Battiti, M. Protasi, Reactive local search for the maximum clique problem, Algorithmica 29 (4) (2001) 610-637.

[9] E. Brickell, V. Wei, Optical orthogonal codes and cyclic block designs, Congr. Numer 58 (1987) 175-192.

[10] M. Brunato, R. Battiti, R-evo: A reactive evolutionary algorithm for the maximum clique problem, IEEE Trans. Evol. Comput. 15 (6) (2011) 770 -782 .

[11] M. Buratti, Recursive constructions for difference matrices and relative difference families, J. Combin. Des. 6 (3) (1998) 165-182.

[12] M. Buratti, K. Momihara, A. Pasotti, New results on optimal $(v, 4,2,1)$ optical orthogonal codes, Des. Codes Cryptogr. 58 (1) (2011) 89-109.

[13] M. Buratti, A. Pasotti, D. Wu, On optimal $(v, 5,2,1)$ optical orthogonal codes, Des. Codes Cryptogr. (2011) 1-23.

[14] W. Chu, C. Colbourn, Optimal $(n, 4,2)$-ooc of small orders, Disc. Math. 279 (1) (2004) 163-172. 
[15] F. Chung, J. Salehi, V. Wei, Optical orthogonal codes: Design, analysis and applications, IEEE Trans. Inf. Theory 35 (3) (1989) 595-604.

[16] C.J. Colbourn, J.H. Dinitz, D.R. Stinson, Applications of combinatorial designs to communications, cryptography, and networking. In: Surveys in Combinatorics 1999, Cambridge University Press, pp. 37-100.

[17] J. De Bonet, C. Isbell, P. Viola, Mimic: Finding optima by estimating probability densities, Advances in neural information processing systems (1997) 424-430.

[18] T. Feng, Y. Chang, L. Ji, Constructions for strictly cyclic 3-designs and applications to optimal oocs with $\lambda=2$, J. Combin. Theory Ser. A 115 (8) (2008) 1527-1551.

[19] X. Geng, J. Xu, J. Xiao, L. Pan, A simple simulated annealing algorithm for the maximum clique problem, Inf. Sciences 177 (22) (2007) 50645071.

[20] A. Grosso, M. Locatelli, F. Croce, Combining swaps and node weights in an adaptive greedy approach for the maximum clique problem, J. Heuristics 10 (2) (2004) 135-152.

[21] J. Håstad, Clique is hard to approximate withinn 1- $\varepsilon$, Acta Math. 182 (1) (1999) 105-142.

[22] K. Katayama, A. Hamamoto, H. Narihisa, An effective local search for the maximum clique problem, Inf. Proc. Letters 95 (5) (2005) 503-511.

[23] W. C. Kwong, P. A. Perrier, and P. R. Prucnal, Performance comparison of asynchronous and synchronous code-division multiple-access techniques for fiber-optical local area networks, IEEE Trans. Commun. 39 (11) (1991) 1625-1634.

[24] E. Marchiori, A simple heuristic based genetic algorithm for the maximum clique problem, in: Proc. ACM Symp. Appl. Comput., Citeseer, 1998, pp. 366-373.

[25] E. Marchiori, Genetic, iterated and multistart local search for the maximum clique problem, Applications of Evolutionary Computing (2002) $112-121$.

[26] P. Ostergard, A fast algorithm for the maximum clique problem, Discrete Applied Mathematics 120 (1) (2002) 197-207.

[27] J. Pardalos, M. Resende, On maximum clique problems in very large graphs, DIMACS series 50 (1999) 119-130.

[28] P. R. Prucnal, M. A. Santoro, and T. R. Fan, Spread spectrum fiberoptic local area network using optical processing, J. Lightwave Technol., 4 (1986) 547-554.

[29] J. Régin, Using constraint programming to solve the maximum clique problem, in: Principles and Practice of Constraint Programming-CP 2003, Springer, 2003, pp. 634-648.

[30] S. Rebennack , M. Oswald, D. Theis, H. Seitz, G. Reinelt, P. M. Pardalos, A branch and cut solver for the maximum stable set problem, Journal of Combinatorial Optimization, 21 (4) (2011) 434-457.

[31] J.A. Salehi, Code division multiple access techniques in optical fiber networks-part I: Fundamental principles, IEEE Trans. Commun. 37 (8) (1989) 824-833.

[32] Q. Wu, J.-K. Hao, A review on algorithms for maximum clique problems, European Journal of Operational Research 242 (3) (2015) 693-709.

[33] Q. Zhang, J. Sun, E. Tsang, An evolutionary algorithm with guided mutation for the maximum clique problem, IEEE Trans. Evol. Comput. 9 (2) (2005) 192-200. 